\title{
Focus on neuro-immune interactions
}

\author{
In this issue, Nature Neuroscience presents a focus on neuro-immune interactions.
}

$\mathrm{T}$ he mammalian nervous system is an elaborate and often fragile structure which is susceptible to damage from injury, pathogen invasion or disease. Such perturbations are known to induce an inflammatory response which often involves infiltration of immune cells and activation of resident effectors, such as microglia. Traditionally, damage-related inflammation was considered to be the only interaction that occurs between the immune system and the 'immunoprivileged' CNS. However, recent evidence suggests that the interplay between these two intricate systems is far more complex than had been previously realized. In this issue, we present a special focus on neuro-immune interactions which highlights some of the most recent and interesting research aimed at understanding the reciprocal relationship between the immune and nervous systems.

Despite being segregated from the rest of the body via the bloodbrain barrier, the CNS still needs to be monitored for infection and/or trauma. A review by Shalina Ousman and Paul Kubes looks at the roles of both the resident sentinels and circulating immune cells in providing immunosurveillance in the CNS. The authors discuss the role of microglial cells in detecting perturbations in the CNS parenchyma, including pathogen infiltration and endogenous disease agents. In addition, they examine recent advances in our understanding of how peripheral leukocytes such as $\mathrm{CD} 4^{+} \mathrm{T}$ cells gain access to the CNS in both health and disease to participate in surveillance and antigen clearance.

As the primary resident immune cells of the nervous system, microglia are responsible for initiating a number of neural responses to insult and disease, including the aforementioned immunosurveillance functions. In their perspective article, Simon Beggs, Tuan Trang and Michael Salter propose that a subset of these multifunctional cells are essential for driving chronic neuropathic pain after peripheral nerve injury. The authors review evidence which suggests that damage to peripheral afferents induces expression of the purinergic receptor P2X4 on microglia and leads to disinhibition of the nociceptive pathways. They also discuss the implications of these findings on the development of potential therapeutics for the amelioration of diseaseor trauma-induced neuropathic pain.

Cell replacement therapies which involve the use of neural stem cells have garnered much attention in recent years as potential mechanisms to drive recovery after CNS injury or disease. In their review, Zaal Kokaia, Gianvito Martino, Michal Schwartz and Olle Lindvall examine the interplay between neural stem and immune cells both in health and in response to pathology. The authors highlight emerging evidence which suggests that immune cells, via cytokine and trophic factor secretion, can influence endogenous neural stem cell proliferation, differentiation and migration. Concurrently, they discuss how engrafted neural stem cells have been shown to influence the initiation and maintenance of inflammatory events while providing insight into how this reciprocal relationship might inform potential therapeutic strategies.

Acute illness, which often results from infection by external pathogens, induces a number of stereotyped symptoms including sleepiness, fever, aches and loss of appetite which are believed to aid the immune system in its battle to control and clear the contagion. On page 1088, Clifford Saper, Andrej Romanovsky and Thomas Scammell review the role of prostaglandins in linking systemic infection with the generation of the 'sickness syndrome'. This review primarily focuses on the targets of prostaglandin signaling and the precise neural circuitry that underlies the genesis of each symptom following peripheral immune activation.

Although neural-immune interactions can often serve to promote healing and recovery after insult, aberrant or unchecked inflammation can also produce detrimental effects in the CNS. One such example is the autoimmune demyelinating disorder, multiple sclerosis. Numerous studies have, with varying degrees of success, sought to gain insight into the pathogenesis and progression of MS using animal models of the disease. In a perspective article on page 1074, Richard Ransohoff describes these various animal models. Illustrating the relative strengths and weaknesses of each, Dr. Ransohoff pays particular attention to which aspects of the human disease each system recapitulates, thus providing insight into the types of biological and pathological questions that each model is suited to address and offering a caution for investigators interested in developing potential therapeutics for this debilitating disease.

While all these articles highlight the impact of the immune system of neural function, Issac Chiu, Christian von Hehn and Clifford Woolf suggest that the inverse may also be true. In a perspective article on page 1063 , these authors postulate that the somatosensory and autonomic nervous systems play an active role in host defense via direct regulation of immunological and inflammatory responses in the periphery. They discuss examples of both positive and negative modulation of immune responses by neural mechanisms and caution that potential treatments aimed at alleviating immune dysfunction may also need to take into account these often overlooked regulatory mechanisms.

The work described by the reviews and perspectives presented in this focus issue has vastly improved our understanding of the intricate and often reciprocal relationship between the neural and immune systems. Once thought to be separate and distinct entities, it is becoming increasingly clear that a number of diverse functions, including endogenous host-defense, disease response and postinfection or -injury repair depend on the activity and interplay of these systems. We hope that these pieces present a coherent picture of the current thought on these topics and will serve to promote further work aimed at understanding these intricate issues and perhaps inform future therapeutics. 